PROCEEDINGS OF THE

AMERICAN MATHEMATICAL SOCIETY

Volume 137, Number 11, November 2009, Pages 3679-3687

S 0002-9939(09)09999-7

Article electronically published on June 22, 2009

\title{
TOWARDS THE CARPENTER'S THEOREM
}

\author{
MARTÍN ARGERAMI AND PEDRO MASSEY
}

(Communicated by Marius Junge)

\begin{abstract}
Let $\mathcal{M}$ be a $\mathrm{II}_{1}$ factor with trace $\tau, \mathcal{A} \subseteq \mathcal{M}$ a masa and $E_{\mathcal{A}}$ the unique conditional expectation onto $\mathcal{A}$. Under some technical assumptions on the inclusion $\mathcal{A} \subseteq \mathcal{M}$, which hold true for any semiregular masa of a separable factor, we show that for elements $a$ in certain dense families of the positive part of the unit ball of $\mathcal{A}$, it is possible to find a projection $p \in \mathcal{M}$ such that $E_{\mathcal{A}}(p)=a$. This shows a new family of instances of a conjecture by Kadison, the so-called "carpenter's theorem".
\end{abstract}

\section{INTRODUCTION}

As is well-known, the Pythagorean Theorem (PT) states that the square of the norm of the sum of two orthogonal vectors is equal to the sum of the squares of the norms of each vector. A converse of the theorem would be the statement that if such an equality occurs, then the two vectors were orthogonal to begin with. Such a result allows a carpenter to check his right-angles by just measuring length, so that's why PT's converse is called the "carpenter's theorem" (CT) by Kadison. In his work [4, 5], he considers extensions of PT and its corresponding converses CT to infinite dimension, getting to the unexpected and striking Theorem 15 in [5] (extended by Arveson in 2]). These generalizations of PT and CT are carried in 4 to the realm of $\mathrm{II}_{1}$ factors, where the PT basically becomes tautological, and the CT becomes the following:

Conjecture (Kadison's carpenter's theorem). Let $\mathcal{A}$ be a masa of the $\mathrm{II}_{1}$ factor $\mathcal{M}$ and let $a \in \mathcal{A}_{1}^{+}$. Then there exists a projection $p \in \mathcal{P}(\mathcal{M})$ such that $E_{\mathcal{A}}(p)=a$, where $E_{\mathcal{A}}$ denotes the trace-preserving conditional expectation onto $\mathcal{A}$.

In the finite dimensional case, the $\mathrm{CT}$ is a particular case of the well-known Schur-Horn theorem. Whether the Schur-Horn theorem extends or not to $\mathrm{II}_{1}$ factors is unknown at the moment (see [1, 3]). In this paper we focus on the CT in $\mathrm{II}_{1}$ factors. Assuming some restrictions on the factor and the masa, which hold true for semiregular masas in separable $\mathrm{II}_{1}$ factors, we show that the statement holds for various dense families. It is worth mentioning here that the statement of the CT (and also of Schur-Horn) is only meaningful in the case of masas, for this would

Received by the editors July 17, 2007.

2000 Mathematics Subject Classification. Primary 46L99; Secondary 46L55.

Key words and phrases. Diagonals of operators, Schur-Horn theorem, conditional expectations.

The first author was supported in part by the Natural Sciences and Engineering Research Council of Canada.

The second author was supported in part by CONICET of Argentina, UNLP, and a PIMS Postdoctoral Fellowship. 
imply the result for any other abelian subalgebra, and also because both statements are likely to fail when the subalgebra considered is not abelian: indeed, CT does not hold for non-abelian subalgebras of $M_{n}(\mathbb{C})$, and so neither does Schur-Horn.

Although our results fail to settle the CT conjecture in full generality, our methods lead us to consider a possible strategy for obtaining the $\mathrm{CT}$ under the conditions we consider for the inclusion $\mathcal{A} \subseteq \mathcal{M}$, as explained at the end of the paper. It is worth noting that these technical conditions hold true for inclusions $\mathcal{A} \subseteq \mathcal{M}$, where $\mathcal{A}$ is semiregular.

\section{Preliminaries}

Throughout the paper $\mathcal{M}$ denotes a $\mathrm{II}_{1}$ factor with normalized faithful normal trace $\tau$. We denote by $\mathcal{M}^{\mathrm{sa}}, \mathcal{M}^{+}, \mathcal{U}_{\mathcal{M}}$, the sets of selfadjoint, positive, and unitary elements of $\mathcal{M}$. By $\mathcal{P}(\mathcal{M})$ we mean the set of projections of $\mathcal{M}$. Given $a \in \mathcal{M}^{\text {sa }}$ we denote its spectral measure by $p^{a}$; thus, $p^{a}(\Delta)$ is the spectral projection associated with a Borel set $\Delta \subset \mathbb{R}$. The characteristic function of the set $\Delta$ is denoted by $\chi_{\Delta}$ and its Lebesgue measure by $m(\Delta)$. The unitary orbit of $a \in \mathcal{M}^{s a}$ is the set $\mathcal{U}_{\mathcal{M}}(a)=\left\{u a u^{*}: u \in \mathcal{U}_{\mathcal{M}}\right\}$.

In 4, Kadison conjectured that if $\mathcal{A} \subseteq \mathcal{M}$ is a masa and $a \in \mathcal{A}_{1}^{+}$i.e., $a \in \mathcal{A}^{+}$ and $0 \leq a \leq 1$, then there exists a projection $p \in \mathcal{P}(\mathcal{M})$ such that $E_{\mathcal{A}}(p)=a$. This conjecture is equivalent to the following assertion: for $p \in \mathcal{P}(\mathcal{M}), a \in \mathcal{A}$,

$$
0 \leq a \leq 1, \tau(a)=\tau(p) \Leftrightarrow a \in E_{\mathcal{A}}\left(\mathcal{U}_{\mathcal{M}}(p)\right) .
$$

Using (11) it can be shown that Kadison's conjecture is a particular case of a more general conjecture (a Schur-Horn theorem in $\mathrm{II}_{1}$ factors), which was stated as an open problem by Arveson and Kadison in 3]. In [1] we proved a weaker version of Arveson-Kadison's conjecture, which restricted to the situation in (1) is

Theorem 2.1. Let $\mathcal{A} \subseteq \mathcal{M}, a \in \mathcal{A}, p \in \mathcal{P}(\mathcal{M})$. Then

$$
0 \leq a \leq 1, \quad \tau(a)=\tau(p) \Leftrightarrow a \in{\overline{E_{\mathcal{A}}\left(\mathcal{U}_{\mathcal{M}}(p)\right)}}^{\text {sot }} .
$$

Note that in (11) the unitary orbit of the projection is already strongly closed (and so norm-closed, too), but the statement in Theorem 2.1] is weaker because it is not clear whether the set on the right-hand side of (11) is already closed in the strong operator topology (a fact that is actually equivalent to Kadison's conjecture by Theorem 2.11).

Matrix units. Given a masa $\mathcal{A}$ in $\mathcal{M}$, we denote by $\mathcal{N}_{\mathcal{A}}$ the normalizer of $\mathcal{A}$ in $\mathcal{M}$, i.e. the subgroup of $\mathcal{U}_{\mathcal{M}}$ given by

$$
\mathcal{N}_{\mathcal{A}}=\left\{u \in \mathcal{U}_{\mathcal{M}}: u^{*} \mathcal{A} u=\mathcal{A}\right\} .
$$

The masa $\mathcal{A}$ is said to be semiregular if $\left(\mathcal{N}_{\mathcal{A}}\right)^{\prime \prime}$ is a factor, and regular (or Cartan) if $\left(\mathcal{N}_{\mathcal{A}}\right)^{\prime \prime}=\mathcal{M}$. Popa shows in [6, Proposition 3.6] that any semiregular masa in a separable type II factor is Cartan in a hyperfinite subfactor. His result implies the following:

Proposition 2.2. If $\mathcal{A} \subset \mathcal{M}$ is a semiregular masa in the separable $\mathrm{II}_{1}$ factor $\mathcal{M}$, then for every $k \in \mathbb{N}$ there exists $\left\{u_{i}^{k}\right\}_{i=1}^{2^{k}} \subset \mathcal{N}_{\mathcal{A}}$ and $\left\{p_{i}^{k}\right\}_{i=1}^{2^{k}} \subset \mathcal{P}(\mathcal{A})$ such that 
$\left\{v_{i j}^{k}\right\}_{i j}$, where $v_{i j}^{k}=u_{i}^{k} p_{1}^{k}\left(u_{j}^{k}\right)^{*}$, is a $2^{k}$-system of matrix units with $v_{j j}^{k}=p_{j}^{k} \in \mathcal{P}(\mathcal{A})$ for $j=1, \ldots, 2^{k}$ and such that

$$
v_{2 i-1,2 j-1}^{k+1}+v_{2 i, 2 j}^{k+1}=v_{i j}^{k}, \quad 1 \leq i, j \leq 2^{k},
$$

and such that the family $\left\{p_{j}^{k}\right\}$ generates all of $\mathcal{A}$.

Matrix units can always be constructed in a $\mathrm{II}_{1}$ factor, but the result in Proposition 2.2 allows one to make "coherent embeddings", in a sense made precise in Corollary 2.3 .

We denote by $\mathcal{D}(n)$ the diagonal subalgebra of $M_{n}(\mathbb{C})$ and by $E_{\mathcal{D}(n)}: M_{n}(\mathbb{C}) \rightarrow$ $\mathcal{D}(n)$ the diagonal compression. We also consider $\phi_{k}: M_{2^{k}}(\mathbb{C}) \rightarrow M_{2^{k+1}}(\mathbb{C})$ to be the unital *-monomorphism $\phi_{k}(A)=I_{2} \otimes A$. Denote by $\left\{e_{i j}^{k}\right\}$ the canonical matrix units in $M_{2^{k}}(\mathbb{C})$.

Corollary 2.3. Let $\left\{p_{j}^{k}\right\},\left\{v_{i j}^{k}\right\}$ be as in Proposition 2.2. Define a family of $*$ monomorphisms $\pi_{k}: M_{2^{k}}(\mathbb{C}) \rightarrow \mathcal{M}$ in the following way: for $a=\left(a_{i j}\right) \in M_{2^{k}}(\mathbb{C})$, let

$$
\pi_{k}(a)=\sum_{i, j} a_{i j} v_{i j}^{k}
$$

Then $\pi_{k}\left(e_{i i}^{k}\right)=p_{i}^{k}$ for $i=1, \ldots, 2^{k}$, and $\pi_{k}=\pi_{k+1} \circ \phi_{k}, \pi_{k} \circ E_{\mathcal{D}\left(2^{k}\right)}=E_{\mathcal{A}} \circ \pi_{k}$, $k \in \mathbb{N}$.

For every $k \in \mathbb{N}$ let $\left\{I_{i}^{k}\right\}_{i=1}^{2^{k}}$ denote the dyadic partition of $[0,1]$ given by $I_{i}^{k}=$ $\left[(i-1) 2^{-k}, i 2^{-k}\right)$.

Remark 2.4. To each family $\left\{\left\{p_{i}^{k}\right\}_{i=1}^{2^{k}}: k \in \mathbb{N}\right\} \subseteq \mathcal{A}$ as in Proposition 2.2 we associate an operator $x$ in the following way. It is easy to see that the sequence of (discrete) positive operators $x_{k}=\sum_{i=1}^{2^{k}} \frac{i}{2^{k}} p_{i}^{k} \in \mathcal{A}^{+}$is non-increasing and bounded. Let $x=\lim _{\mathrm{SOT}} x_{k} \in \mathcal{A}^{+}$. Then, for every $k \in \mathbb{N}$ and $0 \leq i \leq 2^{k}, p^{x}\left(I_{i}^{k}\right)=p_{i}^{k}$. In particular, $\tau \circ p^{x}$ is the Lebesgue measure restricted to $[0,1]$. We say that $x$ is the associated operator to the family $\left\{p_{i}^{k}\right\}$. Notice that the von Neumann subalgebra generated by $x$ coincides with $\mathcal{A}$, since the projections $p_{j}^{k}$ are Borel functional calculus of $x \in \mathcal{A}$.

\section{MAIN RESUlts}

Two subalgebras $\mathcal{A}, \mathcal{B} \subset \mathcal{M}$ are said to be orthogonal [7] in $\mathcal{M}$ if $E_{\mathcal{A}}(\mathcal{B}) \subset \mathbb{C} I$.

Definition 3.1. We say that a masa $\mathcal{A} \subset \mathcal{M}$ is totally complementable if for every projection $p \in \mathcal{A}$, the masa $p \mathcal{A}$ in $p \mathcal{M} p$ admits a diffuse orthogonal subalgebra.

In what follows we shall say that $a \in \mathcal{M}^{+}$is discrete if there exists a sequence of mutually orthogonal projections $\left\{q_{k}\right\}_{k \in \mathbb{N}} \subset \mathcal{M}$ and a sequence of uniformly bounded complex numbers $\left\{\alpha_{k}\right\}_{k \in \mathbb{N}}$ such that $a=\sum_{k} \alpha_{k} q_{k}$ (where the convergence is in the $\|\cdot\|_{1}$-norm). Note that we can always assume that $\alpha_{k} \neq \alpha_{j}$ if $k \neq j$.

Theorem 3.2 (Carpenter's theorem for discrete operators). If $\mathcal{A}$ is a totally complementable masa in the $\mathrm{II}_{1}$ factor $\mathcal{M}$, then for every discrete $a \in(\mathcal{A})_{1}^{+}$there exists a projection $p \in \mathcal{M}$ such that $E_{\mathcal{A}}(p)=a$. 
Proof. Assume $\mathcal{B} \subset \mathcal{M}$ is a subalgebra orthogonal to $\mathcal{A}$. For any $\alpha \in[0,1]$, there exists a projection $q \in \mathcal{B}$ with $\tau(q)=\alpha$. Since $\mathcal{A}$ and $\mathcal{B}$ are orthogonal, $E_{\mathcal{A}}(q)=$ $\tau\left(E_{\mathcal{A}}(q)\right)=\tau(q)=\alpha$.

Now let $p \in \mathcal{A}$ be a projection; then $p \mathcal{A}$ is a masa in $p \mathcal{M} p$, so it admits an orthogonal subalgebra $\mathcal{B}_{p}$. By the first paragraph, there exists a projection $q \in$ $\mathcal{B}_{p} \subset p \mathcal{M} p$ with $E_{p \mathcal{A}}(q)=\alpha p$. Since $q \in p \mathcal{M} p$, in particular $q=p q$. So

$$
E_{\mathcal{A}}(q)=E_{\mathcal{A}}(p q)=p E_{\mathcal{A}}(q)=E_{p \mathcal{A}}(q)=\alpha p .
$$

Now let $a=\sum_{k} \alpha_{k} p_{k} \in \mathcal{A}$, where $\left\{p_{k}\right\}_{k \in \mathbb{N}}$ is a sequence of mutually orthogonal projections in $\mathcal{M}$ and $\left\{\alpha_{k}\right\}_{k \in \mathbb{N}}$ is a sequence of uniformly bounded numbers such that $\alpha_{k} \neq \alpha_{j}$ for $k \neq j$. Since $a \in(\mathcal{A})_{1}^{+}$, for each $k \in \mathbb{N}$ we have that $p_{k} \in \mathcal{A}$ (since we can recover these projections as Borel functional calculus of $a$ ) and $0 \leq \alpha_{k} \leq 1$. For each $k \in \mathbb{N}$ apply the first part of the proof to get a projection $q_{k} \in \mathcal{M}^{+}$such that $E_{\mathcal{A}}\left(q_{k}\right)=\alpha_{k} p_{k}, q_{k} \leq p_{k}$. Thus, the operator $q=\sum_{k} q_{k} \in \mathcal{M}$ is a projection such that $E_{\mathcal{A}}(q)=\sum_{k} \alpha_{k} p_{k}$.

Remarks 3.3. (i) The conditions in Theorem 3.2 are satisfied by a Cartan masa of the hyperfinite $\mathrm{II}_{1}$ factor, and so by any semiregular masa in a separable $\mathrm{II}_{1}$ factor, since it is Cartan in an intermediate hyperfinite subfactor [6, Proposition 3.6].

(ii) Because in general there is no clear "coherent" way of constructing the projections $q_{k}$ in the previous proof, we would not expect such an argument to be useful to prove the general case of the carpenter's theorem.

(iii) Under the conditions of Theorem [3.2, it follows in particular that there exists a projection $p \in \mathcal{A}$ such that

$$
E_{\mathcal{A}}(p)=\frac{1}{\sqrt{2}} I .
$$

Remarkably, it seems hard to prove even this particular case of Kadison's conjecture in the general case of an arbitrary $\mathrm{II}_{1}$ factor and a masa $\mathcal{A} \subseteq \mathcal{M}$.

In the remainder of the paper, given a semiregular masa $\mathcal{A}$ of the separable $\mathrm{II}_{1}$ factor $\mathcal{M}$, we will prove the carpenter's theorem for some non-discrete operators, namely piecewise linear functional calculus of $x$, the associated operator of a family of projections considered in Remark 2.4

We begin by defining the following sequence of unitary matrices $\left(W_{n}\right)_{n}$ :

$$
W_{1}=\left(\begin{array}{cccc}
1 & 0 & 0 & 0 \\
0 & \frac{1}{\sqrt{2}} & -\frac{1}{\sqrt{2}} & 0 \\
0 & \frac{1}{\sqrt{2}} & \frac{1}{\sqrt{2}} & 0 \\
0 & 0 & 0 & 1
\end{array}\right), \quad W_{n+1}=W_{n} \otimes I_{2}=\left(\begin{array}{cc}
W_{n} & 0 \\
0 & W_{n}
\end{array}\right)=\bigoplus_{j=1}^{2^{n}} W_{1}
$$

Lemma 3.4. Let $A \in M_{2^{k}}(\mathbb{C})$. Put $A(1)=A, A(n+1)=W_{k+n-1}\left(I_{2} \otimes\right.$ $A(n)) W_{k+n-1}^{*}$. Then there exists $\lambda<1$, independent of $A, k$ and $n$ such that

$$
\frac{1}{2}\left\|A(n+1)-I_{2} \otimes A(n)\right\|_{2}^{2} \leq \lambda\left\|A(n)-I_{2} \otimes A(n-1)\right\|_{2}^{2} .
$$

Proof. Let $k \geq 1$ and $n \geq 2$. We can consider $A(n-1)$ as a block matrix with $2 \times 2$ blocks, i.e. $A(n-1)=\left(A_{i j}\right)_{i j}$, where $A_{i j} \in M_{2}(\mathbb{C})$ for $1 \leq i, j \leq 2^{(k+n-3)}$. It is easy to verify that

$$
I_{2} \otimes A(n-1)=\left(I_{2} \otimes A_{i j}\right)_{i j} \text { and } A(n)=\left(W_{1}\left(I_{2} \otimes A_{i j}\right) W_{1}^{*}\right)_{i j}=\left(A_{i j}(2)\right)_{i j} .
$$


So in particular we have that

$$
\left\|A(n)-I_{2} \otimes A(n-1)\right\|_{2}^{2}=\sum_{i, j=1}^{2^{(k+n-3)}}\left\|A_{i j}(2)-I_{2} \otimes A_{i j}\right\|_{2}^{2} .
$$

Similarly we see that $A(n+1)=\left(A_{i j}(3)\right)_{i j}, \quad$ for $1 \leq i, j \leq 2^{k+n-3}$ and

$$
\left\|A(n+1)-I_{2} \otimes A(n)\right\|_{2}^{2}=\sum_{i, j=1}^{2^{(k+n-3)}}\left\|A_{i j}(3)-I_{2} \otimes A_{i j}(2)\right\|_{2}^{2} .
$$

So, from (3) and (4) we see that it is enough to prove that there exists $0<\lambda<1$ (independent of $A, k$ and $n$ ) such that for every $1 \leq i, j \leq 2^{k+n-3}$,

$$
\frac{1}{2}\left\|A_{i j}(3)-I_{2} \otimes A_{i j}(2)\right\|_{2}^{2} \leq \lambda\left\|A_{i j}(2)-I_{2} \otimes A_{i j}\right\|_{2}^{2} .
$$

We show that such an inequality holds for any $2 \times 2$ matrix $B=\left(b_{i j}\right)_{i j} \in M_{2}(\mathbb{C})$. By straightforward computations,

$$
B(2)=W_{1}\left(I_{2} \otimes B\right) W_{1}^{*}=\left(\begin{array}{cccc}
b_{11} & \frac{-b_{12}}{\sqrt{2}} & \frac{b_{12}}{\sqrt{2}} & 0 \\
\frac{-b_{21}}{\sqrt{2}} & \frac{b_{11}+b_{22}}{2} & \frac{b_{11}-b_{22}}{2} & \frac{b_{12}}{\sqrt{2}} \\
\frac{b_{21}}{\sqrt{2}} & \frac{b_{11}-b_{22}}{2} & \frac{b_{11}+b_{22}}{2} & \frac{b_{12}}{\sqrt{2}} \\
0 & \frac{b_{21}}{\sqrt{2}} & \frac{b_{21}}{\sqrt{2}} & b_{22}
\end{array}\right)
$$

and so

$$
\left\|B(2)-I_{2} \otimes B\right\|_{2}^{2}=(4-2 \sqrt{2})\left(\left|b_{12}\right|^{2}+\left|b_{21}\right|^{2}\right)+\left|b_{11}-b_{22}\right|^{2} .
$$

Thus, if we consider $B(2)=\left(B_{i j}\right)_{i j}$ as a $2 \times 2$ block matrix, where $B_{i j} \in M_{2}(\mathbb{C})$, we can use the previous calculation with each of these four matrices and get

(6) $\frac{1}{2}\left\|B(3)-I_{2} \otimes B(2)\right\|_{2}^{2}=\frac{1}{2}\left((4-2 \sqrt{2})\left(\left|b_{12}\right|^{2}+\left|b_{21}\right|^{2}\right)+\left(\frac{5}{2}-\sqrt{2}\right)\left|b_{11}-b_{22}\right|^{2}\right)$.

Writing $\frac{5}{2}-\sqrt{2}=1+\left(\frac{3}{2}-\sqrt{2}\right)$ and using (5) and (6) we get that

$$
\frac{1}{2} \frac{\left\|B(3)-I_{2} \otimes B(2)\right\|_{2}^{2}}{\left\|W_{1}\left(I_{2} \otimes B\right) W_{1}^{*}-I_{2} \times B\right\|_{2}^{2}} \leq \frac{1}{2}\left(1+\frac{3}{2}-\sqrt{2}\right)<1 .
$$

In what follows we denote by $\left\{f_{i}^{k}\right\}_{i=1}^{2^{k}}$ the rank-one projections associated with the elements of the canonical basis of $\mathbb{C}^{2^{k}}$, that is, $f_{i}^{k}=e_{i i}^{k}$.

Lemma 3.5. Let $n \in \mathbb{N}$ and $A \in M_{2^{k}}(\mathbb{C})$. Then, with the notation of Lemma 3.4.

(i) $E_{\mathcal{D}\left(2^{k+n}\right)}(A(n+1))=E_{\mathcal{D}\left(2^{k+n}\right)}\left(W_{k+n-1}\left(I_{2} \otimes E_{\mathcal{D}\left(2^{k+n-1}\right)}(A(n))\right) W_{k+n-1}^{*}\right)$.

(ii) If $A$ is diagonal and $B=W_{k-1} A W_{k-1}^{*}$, then

$$
B_{i i}= \begin{cases}A_{i i} & \text { if } i=4 h \text { or } i=4 h-3, \\ \frac{1}{2}\left(A_{4 h-1,4 h-1}+A_{4 h-2,4 h-2}\right) & \text { if } i=4 h-1 \text { or } i=4 h-2 .\end{cases}
$$


(iii) If $E_{\mathcal{D}\left(2^{k}\right)}(A)=\sum_{\ell=1}^{2^{k}} d_{\ell} f_{\ell}^{k}$, then

$$
E_{\mathcal{D}\left(2^{k+n-1}\right)}(A(n))=\sum_{\ell=1}^{2^{k-1}} \sum_{h=1}^{2^{n-1}} \gamma_{\ell, h-1}^{n} f_{2^{n}(\ell-1)+2 h-1}^{k+n-1}+\gamma_{\ell, h}^{n} f_{2^{n}(\ell-1)+2 h}^{k+n-1}
$$

where

$$
\gamma_{\ell, h}^{n}=d_{2 \ell-1}+\frac{h}{2^{n-1}}\left(d_{2 \ell}-d_{2 \ell-1}\right)
$$

Proof. To prove (i) let $k, n \geq 1$ and consider the block representations $A(n)=$ $\left(A_{i j}\right)_{i, j=1}^{2^{k+n-2}}$, where $A_{i j} \in M_{2}(\mathbb{C})$. Then $I_{2} \otimes A(n)=\left(I_{2} \otimes A_{i j}\right)_{i j=1}^{2^{k+n-2}}$ and

$$
A(n+1)=W_{k+n-1}\left(I_{2} \otimes A(n)\right) W_{k+n-1}^{*}=\left(W_{1}\left(I_{2} \otimes A_{i j}\right) W_{1}^{*}\right)_{i j=1}^{2^{k+n-2}}
$$

with respect to the previous block representation. Hence, to study the diagonal of $A(n+1)$ we can restrict our attention to the diagonal blocks $W_{1}\left(I_{2} \otimes A_{i i}\right) W_{1}^{*} \in$ $M_{4}(\mathbb{C})$, for $i=1, \ldots, 2^{k+n-2}$. Straightforward computations show that

$$
E_{\mathcal{D}(4)}\left(W_{1}\left(I_{2} \otimes A_{i i}\right) W_{1}^{*}\right)=E_{\mathcal{D}(4)}\left(W_{1} E_{\mathcal{D}(4)}\left(I_{2} \otimes A_{i i}\right) W_{1}^{*}\right)
$$

from which (i) follows, after noting that $E_{\mathcal{D}(4)}\left(I_{2} \otimes B\right)=I_{2} \otimes E_{\mathcal{D}(2)}(B)$ for any $B \in M_{2}(\mathbb{C})$.

The proof of (iil) is straightforward.

We prove (iii) by induction. The case $n=1$ follows from the definitions, and hence we omit it. Now, assume that (iii) holds for $A(n)$. Then

$$
\begin{aligned}
I_{2} \otimes E_{\mathcal{D}\left(2^{k+n-1}\right)}(A(n))= & \sum_{\ell=1}^{2^{k-1}} \sum_{h=1}^{2^{n-1}} \gamma_{\ell, h-1}^{n} I_{2} \otimes f_{2^{n}(\ell-1)+2 h-1}^{k+n-1} \\
& +\gamma_{\ell, h}^{n} I_{2} \otimes f_{2^{n}(\ell-1)+2 h}^{k+n-1} \\
= & \sum_{\ell=1}^{2^{k-1}} \sum_{h=1}^{2^{n-1}} \gamma_{\ell, h-1}^{n}\left(f_{(\ell-1) 2^{n+1}+4 h-3}^{k+n}+f_{(\ell-1) 2^{n+1}+4 h-2}^{k+n}\right) \\
& +\gamma_{\ell, h}^{n}\left(f_{(\ell-1) 2^{n+1}+4 h-1}^{k+n}+f_{(\ell-1) 2^{n+1}+4 h}^{k+n}\right) .
\end{aligned}
$$

Using (iii) and the relations

$$
\gamma_{\ell, h}^{n}=\gamma_{\ell, 2 h}^{n+1}, \quad \frac{1}{2}\left(\gamma_{\ell, h-1}^{n}+\gamma_{\ell, h}^{n}\right)=\gamma_{\ell, 2 h-1}^{n+1},
$$

we have 


$$
\begin{aligned}
& E_{\mathcal{D}\left(2^{k+n}\right)}(A(n+1))=E_{\mathcal{D}\left(2^{k+n}\right)}\left(W_{k+n-1}\left(I_{2} \otimes E_{\mathcal{D}\left(2^{k+n-1}\right)}(A(n))\right) W_{k+n-1}^{*}\right) \\
& =\sum_{\ell=1}^{2^{k-1}} \sum_{h=1}^{2^{n-1}} \gamma_{\ell, h-1}^{n} f_{(\ell-1) 2^{n+1}+4 h-3}^{k+n} \\
& +\frac{1}{2}\left(\gamma_{\ell, h-1}^{n}+\gamma_{\ell, h}^{n}\right) f_{(\ell-1) 2^{n+1}+4 h-2}^{k+n} \\
& +\frac{1}{2}\left(\gamma_{\ell, h-1}^{n}+\gamma_{\ell, h}^{n}\right) f_{(\ell-1) 2^{n+1}+4 h-1}^{k+n}+\gamma_{\ell, h}^{n} f_{(\ell-1) 2^{n+1}+4 h}^{k+n} \\
& =\sum_{\ell=1}^{2^{k-1}} \sum_{h=1}^{2^{n-1}} \gamma_{\ell, 2 h-2}^{n+1} f_{(\ell-1) 2^{n+1}+4 h-3}^{k+n}+\gamma_{\ell, 2 h-1}^{n+1} f_{(\ell-1) 2^{n+1}+4 h-2}^{k+n} \\
& +\gamma_{\ell, 2 h-1}^{n+1} f_{(\ell-1) 2^{n+1}+4 h-1}^{k+n}+\gamma_{\ell, 2 h}^{n+1} f_{(\ell-1) 2^{n+1}+4 h}^{k+n} \\
& =\sum_{\ell=1}^{2^{k-1}} \sum_{h=1}^{2^{n}} \gamma_{\ell, h-1}^{n+1} f_{2^{n}(\ell-1)+2 h-1}^{k+n}+\gamma_{\ell, h}^{n+1} f_{2^{n}(\ell-1)+2 h}^{k+n} .
\end{aligned}
$$

Theorem 3.6 (Carpenter's theorem for some non-discrete operators). Let $\mathcal{M}$ be a separable $\mathrm{II}_{1}$ factor and let $x \in \mathcal{A}^{+}$be the associated operator to a family $\left\{p_{i}^{k}\right\}$ of projections in a semiregular masa $\mathcal{A}$ in $\mathcal{M}$. If $A \in M_{2^{k}}(\mathbb{C})$, then the sequence $\left(a_{n}\right)_{n \in \mathbb{N}} \subseteq \mathcal{M}$ given by $a_{1}=\pi_{k}(A)$ and

$$
a_{n+1}=\pi_{k+n}(A(n+1))=\pi_{k+n}\left(W_{n+k-1}\right) \pi_{k+n}(A(n)) \pi_{k+n}\left(W_{n+k-1}\right)^{*}
$$

converges strongly to an operator $a \in \mathcal{M}$. Moreover, we have that

(i) if $A$ is a projector (resp. selfadjoint, positive), then so is $a$;

(ii) if $A_{j j}=d_{j}$ and $f:[0,1] \rightarrow \mathbb{C}$ is the piecewise linear function given by

$$
\begin{gathered}
f(t)=d_{2 j-1}+2^{k-1}\left(t-\frac{j-1}{2^{k-1}}\right)\left(d_{2 j}-d_{2 j-1}\right), \quad t \in\left[\frac{j-1}{2^{-(k-1)}}, \frac{j}{2^{-(k-1)}}\right), \\
j=1, \ldots, 2^{k-1}, \text { then } E_{\mathcal{A}}(a)=f(x) ;
\end{gathered}
$$

(iii) if $B \in M_{2^{k}}$ and $b=\lim _{n} \pi_{n+k-1}(B(n))$, then $\|b-a\|_{2}^{2}=\frac{1}{2^{k}}\|B-A\|_{2}^{2}$.

Proof. Using Corollary 2.3. Lemma 3.4 and the fact that if $C \in M_{2^{k}}(\mathbb{C})$ then $\left\|\pi_{k+n-1}(C)\right\|_{2}^{2}=2^{-(k+n-1)}\|C\|_{2}^{2}$, we have

$$
\left\|a_{n+1}-a_{n}\right\|_{2}^{2} \leq \lambda\left\|a_{n}-a_{n-1}\right\|_{2}^{2}
$$

with $0<\lambda<1$, independent of $A, k$ and $n$. Then the sequence $\left\{a_{n}\right\}$ converges in $\|\cdot\|_{2}$ to an operator $a \in \mathcal{M}$. We now prove the remaining items.

(i) If $A$ is a projector (resp. selfadjoint, positive), then so is $A(n)$, for each $n$. Since every $\pi_{n}$ is a $*$-representation, $\pi_{n+k-1}(A(n))$ inherits the properties from $A$, and any of the three properties passes to the $\|\cdot\|_{2}$-limit.

(ii) By Lemmas 2.3 and 3.5 .

$$
\begin{aligned}
E_{\mathcal{A}}\left(a_{n}\right) & =E_{\mathcal{A}}\left(\pi_{k+n-1}(A(n))\right)=\pi_{k+n-1}\left(E_{\mathcal{D}\left(2^{k+n-1}\right)}(A(n))\right) \\
& =\sum_{\ell=1}^{2^{k-1}} \sum_{h=1}^{2^{n-1}} \gamma_{\ell, h-1}^{n} p_{2^{n}(\ell-1)+2 h-1}^{k+n-1}+\gamma_{\ell, h}^{n} p_{2^{n}(\ell-1)+2 h}^{k+n-1} .
\end{aligned}
$$


If we consider the discrete operators $x_{n}$ as defined in Remark 2.4 then

$$
\begin{aligned}
& x_{k+n-1}=\sum_{i=1}^{2^{k+n-1}} \frac{i}{2^{k+n-1}} p_{i}^{k+n-1} \\
& =\sum_{\ell=1}^{2^{k-1}} \sum_{h=1}^{2^{n-1}} \frac{2^{n}(\ell-1)+2 h-1}{2^{k+n-1}} p_{2^{n}(\ell-1)+2 h-1}^{k+n-1}+\frac{2^{n}(\ell-1)+2 h}{2^{k+n-1}} p_{2^{n}(\ell-1)+2 h}^{k+n-1} .
\end{aligned}
$$

It is easy to check that

$$
\frac{\ell-1}{2^{k-1}} \leq \frac{2^{n}(\ell-1)+2 h-1}{2^{k+n-1}}<\frac{2^{n}(\ell-1)+2 h}{2^{k+n-1}}<\frac{\ell}{2^{k-1}},
$$

and, if $\gamma_{\ell, h}^{n}$ are as in the statement of Lemma 3.5, then

$$
\begin{aligned}
f\left(\frac{2^{n}(\ell-1)+2 h-1}{2^{k+n-1}}\right) & =\gamma_{\ell, h-1}^{n}+\frac{1}{2^{n}}\left(d_{2 \ell}-d_{2 \ell-1}\right), \\
f\left(\frac{2^{n}(\ell-1)+2 h}{2^{k+n-1}}\right) & =\gamma_{\ell, h-1}^{n} .
\end{aligned}
$$

So

$$
\begin{aligned}
f\left(x_{k+n-1}\right) & =\sum_{\ell=1}^{2^{k-1}} \sum_{h=1}^{2^{n-1}}\left(\gamma_{\ell, h-1}^{n}+\frac{1}{2^{n}}\left(d_{2 \ell}-d_{2 \ell}\right)\right) p_{2^{n}(\ell-1)+2 h-1}^{k+n-1} \\
& +\gamma_{\ell, h-1}^{n} p_{2^{n}(\ell-1)+2 h}^{k+n-1} \\
& =E_{\mathcal{A}}\left(a_{n}\right)+\sum_{\ell=1}^{2^{k-1}} \sum_{h=1}^{2^{n-1}} \frac{1}{2^{n}}\left(d_{2 \ell}-d_{2 \ell-1}\right) p_{2^{n}(\ell-1)+2 h-1}^{k+n-1} .
\end{aligned}
$$

Thus, letting $d=\max \left\{d_{i}\right\} \leq\|A\|$,

$$
\left\|E_{\mathcal{A}}\left(a_{n}\right)-f\left(x_{k+n-1}\right)\right\|=\left\|\sum_{\ell=1}^{2^{k-1}} \sum_{h=1}^{2^{n-1}} \frac{1}{2^{n}}\left(d_{2 \ell}-d_{2 \ell-1}\right) p_{2^{n}(\ell-1)+2 h-1}^{k+n-1}\right\| \leq \frac{d}{2^{n}} .
$$

Since $a_{n} \stackrel{\|\cdot\|_{2}}{\longrightarrow} a, x_{n} \stackrel{\|\cdot\|_{2}}{\longrightarrow} x, E_{\mathcal{A}}$ is normal, and $f$ is continuous off a set of Lebesgue measure 0 (see Remark 2.4), we get $E_{\mathcal{A}}\left(a_{n}\right) \stackrel{\|\cdot\|_{2}}{\longrightarrow} E_{\mathcal{A}}(a), f\left(x_{n}\right) \stackrel{\|\cdot\|_{2}}{\longrightarrow} f(x)$, and so $E_{\mathcal{A}}(a)=f(x)$.

(iii) Note that $\left\|I_{2} \otimes A\right\|_{2}^{2}=2\|A\|_{2}^{2}$. Then we have

$$
\begin{aligned}
& \left\|\pi_{n+k-1}(B(n))-\pi_{n+k-1}(A(n))\right\|_{2}^{2}=\frac{1}{2^{n+k-1}}\|B(n)-A(n)\|_{2}^{2} \\
& =\frac{1}{2^{n+k-1}} \| W_{k+n-2}\left(I_{2} \otimes(B(n-1)-A(n-1)) W_{k+n-2} \|_{2}^{2}\right. \\
& =\frac{1}{2^{n+k-2}}\|B(n-1)-A(n-1)\|_{2}^{2} \\
& \vdots \\
& =\frac{1}{2^{k}}\|B-A\|_{2}^{2} .
\end{aligned}
$$

By continuity,

$$
\|b-a\|_{2}^{2}=\frac{1}{2^{k}}\|B-A\|_{2}^{2}
$$


The continuity property in (iii) suggests a possible strategy for solving Kadison's conjecture in this setting: using the previous notation, let $g(x) \in \mathcal{A}$ for $g \in \mathrm{L}^{\infty}([0,1]), 0 \leq g \leq 1$, and for $k \in \mathbb{N}$, let $g_{k}=\sum_{i=1}^{2^{k}} g_{i, k} \chi_{I_{i}^{k}}$ be a sequence of dyadic discrete functions, $0 \leq g_{k} \leq 1, \int_{0}^{1} g_{k}(t) d t=2^{-k} m(k)$ for some $m(k) \in \mathbb{N}$ and such that it converges to $g$ in $\mathrm{L}^{2}([0,1])$. Then, if we were able to construct a sequence of projection matrices $A_{k} \in M_{2^{k}}(\mathbb{C})$ such that

$$
\mathcal{D}_{2^{k}}\left(A_{k}\right)=\sum_{i=1}^{2^{k}} g_{i, k} f_{i}^{k} \quad \text { and } \quad \limsup _{k} \frac{1}{2} \frac{\left\|A_{k+1}-I_{2} \otimes A_{k}\right\|_{2}^{2}}{\left\|A_{k}-I_{2} \otimes A_{k-1}\right\|_{2}^{2}}<1,
$$

then, denoting by $a_{k}=\lim _{n} \pi_{k+n}\left(A_{k}\right)$, we would have that

$$
a_{k} \underset{k}{\stackrel{\|\|_{2}}{\longrightarrow}} a, \quad E_{\mathcal{A}}\left(a_{k}\right) \underset{k}{\stackrel{\|\|_{2}}{\longrightarrow}} g(x)
$$

since by (7), $\left\{a_{k}\right\}_{k}$ would be a Cauchy sequence of projections in $\|\cdot\|_{2}$. Hence $a \in$ $\mathcal{M}^{+}$would be a projection such that $E_{\mathcal{A}}(a)=g(x)$ for an arbitrary $g \in \mathrm{L}^{\infty}([0,1])$, $0 \leq g \leq 1$.

\section{ACKNOWLEDGEMENTS}

The second-named author wishes to thank D. Farenick and the Department of Mathematics and Statistics at the University of Regina for the warm hospitality received during his stay. We would also like to thank R. Sasyk and S. White for useful comments.

\section{REFERENCES}

[1] M. Argerami and P. Massey, A Schur-Horn theorem in II factors, Indiana Univ. Math. J., 56 (2007), no. 5, 2051-2060. MR2359722 (2008m:46120)

[2] W. Arveson, Diagonals of normal operators with finite spectrum, Proc. Natl. Acad. Sci. USA 104 (2007), no. 4, 1152-1158. MR2303566 (2008f:47027)

[3] W. Arveson and R. Kadison, Diagonals of self-adjoint operators, in D. R. Larson, D. Han, P. E. T. Jorgensen, editors, Operator theory, operator algebras and applications, Contemp. Math. Amer. Math. Soc., 2006. MR2277215 (2007k:46116)

[4] R. Kadison, The Pythagorean Theorem: I. The finite case, Proc. Natl. Acad. Sci. USA 99 (2002), no. 7, 4178-4184. MR1895747 (2003e:46108a)

[5] R. Kadison, The Pythagorean Theorem: II. The infinite discrete case, Proc. Natl. Acad. Sci. USA 99 (2003), no. 8, 5217-5222. MR1896498(2003e:46108b)

[6] S. Popa, On a problem of R.V. Kadison on maximal abelian *-subalgebras in factors, Inv. Math. 65 (1981), 269-281. MR641131 (83g:46056)

[7] S. Popa, Orthogonal pairs of *-subalgebras in finite von Neumann algebras, J. Operator Theory 9 (1983), no. 2, 253-268. MR703810 (84h:46077)

[8] A. Sinclair and R. Smith, Finite von Neumann algebras and masas, Cambridge University Press, Cambridge, 2008. MR2433341

Department of Mathematics, University of Regina, Regina Saskatchewan, Canada

E-mail address: argerami@math.uregina.ca

Departamento de Matemática, Universidad Nacional de la Plata and instituto Argentino de Matemática-conicet, Argentina

E-mail address: massey@mate.unlp.edu.ar 